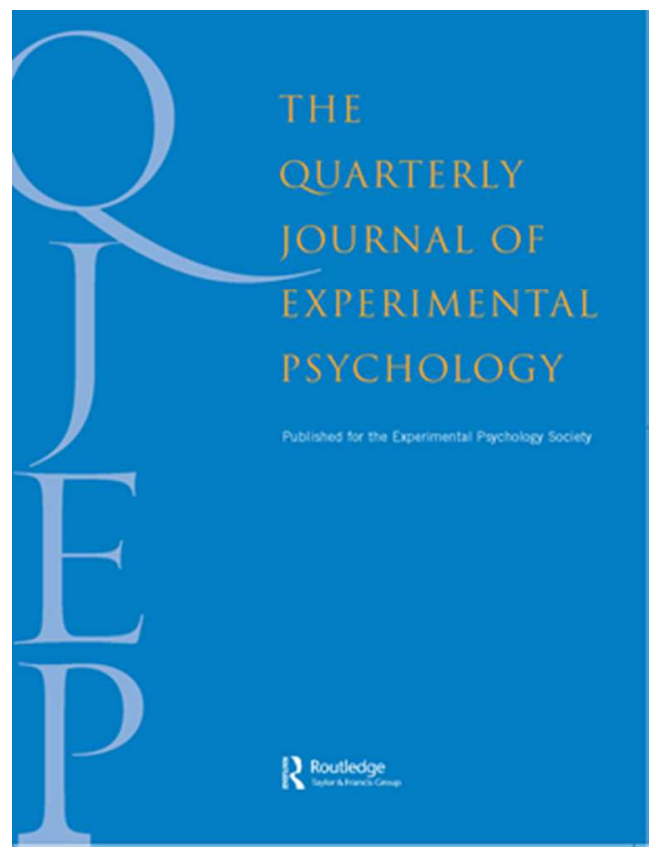

\title{
Relative prediction error and protection from attentional blink in human associative learning
}

\begin{tabular}{|r|l|}
\hline Journal: & Quarterly Journal of Experimental Psychology \\
\hline Manuscript ID: & QJE-STD 13-348.R3 \\
\hline Manuscript Type: & Standard Article \\
\hline Date Submitted by the Author: & n/a \\
\hline Complete List of Authors: & $\begin{array}{l}\text { Glautier, Steven; Southampton University, School of Psychology } \\
\text { Shih, Shui-I; University of Southampton, Psychology }\end{array}$ \\
\hline Keywords: & $\begin{array}{l}\text { associative learning, attentional blink, predictive validity, relative error, } \\
\text { absolute error, prediction error }\end{array}$ \\
\hline \multicolumn{2}{|l}{} \\
\hline
\end{tabular}

\section{SCHOLARONEm}

Manuscripts 


\author{
Relative prediction error and protection from attentional blink \\ in human associative learning \\ Steven Glautier and Shui-1 Shih \\ Psychology, Southampton University
}

Correspondence to:

Steven Glautier

Psychology

University of Southampton

Southampton, SO17 1BJ

United Kingdom

E-mail: spg@,soton.ac.uk

Tel: (+44) 02380592589 


\begin{abstract}
The relationship between predictive learning and attentional processing was investigated in two experiments. During a learning procedure participants viewed rapid serial visual presentation (RSVP) of stimuli in the context of a choice-reaction-time (CRT) task. Salient stimuli in the RSVP streams were either predictive or non-predictive for the outcome of the CRT task.

Following this procedure we measured attentional blink $(\mathrm{AB})$ to the predictive and nonpredictive stimuli. In Experiment 1, despite the use of a large sample and checks demonstrating the validity of the learning procedure and the $\mathrm{AB}$ measure, we did not observe reduced $\mathrm{AB}$ for predictive stimuli. In contrast, in Experiment 2, where the predictive stimuli occurred alongside salient non-predictive comparison stimuli, we did find less $\mathrm{AB}$ for predictive than for nonpredictive stimuli. Our results support an attentional model of learning in which relative prediction error is used to increase learning rates for good predictors and reduce learning rates for poor predictors (Mackintosh, 1975) and provide confirmation of the AB learning effect originally reported by Livesey, Harris, and Harris (2009).
\end{abstract}

Keywords: associative learning, attentional blink, predictive validity, prediction error, relative error, absolute error, replication

Word count: 9513 (body) 10848 (all) 


\section{Relative prediction error and protection from attentional blink}

\section{in human associative learning}

Several models of learning suggest that learning, a central process of association formation, is accompanied by changes in attention to task relevant cues. However, models do not usually characterize what attention entails beyond a change in a learning rate parameter in a mathematical model (e.g. Le Pelley, 2004; Mackintosh, 1975; Pearce \& Hall, 1980). The current paper follows-up a study by Livesey, Harris, and Harris (2009) which showed reduced attentional blink $(\mathrm{AB})$ to stimuli that had predictive value in a choice-reaction-time (CRT) task. This $\mathrm{AB}$ learning effect demonstrated a correspondence between $\mathrm{AB}$ and the learning rate changes described in Mackintosh's (1975) model of associative learning, making concrete a link between an abstract mathematical model and a psychological process. Within the associative learning literature, Mackintosh's (1975) model has frequently been considered incompatible with Pearce and Hall's (1980) model. Both models make use of the concept of prediction error in terms of the difference between the outcome of a learning trial and an expectation derived from the associative strength of the conditioned stimuli (CSs) that are present on that trial. On each trial, the Mackintosh model adjusts learning rates according to relative prediction error whereas the Pearce-Hall model makes use of absolute prediction error. In the Mackintosh model, the learning rate for CSs that are best predictors within a learning task increases, hence these are considered to receive more attention. In contrast, in the Pearce-Hall model, good predictors have their learning rates reduced, hence are considered to receive less attention (see Figure 1 and penultimate paragraph of this introduction for further explanation). In fact both types of prediction error seem to be important, leading to the development of hybrid attentional models (Le Pelley, 2004; Pearce \& Mackintosh, 2010). In our analysis of the experiments to be reported 
below we suggest that the strength of the AB learning effect can be increased by increasing the salience of relative prediction error. Furthermore the AB learning effect gives rise to a particular psychological interpretation of the learning rate changes described by the Mackintosh model. This interpretation suggests an increase in the top-down salience of predictive stimuli that facilitates their entry to, and consolidation in, working memory (Shih, 2008).

To illustrate why we consider the AB learning effect an important advance worthy of replication and further exploration we briefly describe some previous work aiming to differentiate the Mackintosh and Pearce-Hall views of attention in associative learning. Some studies inferred attentional change using speed of learning as an indirect measure of attention with more rapid learning implying increased attention, but did not give any further information about the characteristics of any attentional change (see also Le Pelley, Vadillo, \& Luque, 2013). This approach was used by, for example, Durlach and Mackintosh (1986) to study intradimensional and extra-dimensional shift learning. In this study pigeons were trained in a transfer-test consisting of a series of color discrimination reversals. Prior to the transfer-test they had been pre-trained with another discrimination reversal problem. For one group (the intradimensional shift group) this pre-training involved two different colors whereas for the other group (the extra-dimensional shift group) the pre-training involved discrimination reversals in which line orientations rather than colors had been used as discriminative stimuli. Pre-training with the color-based discrimination reversal resulted in better performance in the color-based transfer-test than did pre-training with the line orientations. They argued that the pre-training increased attention to color cues, consistent with Mackintosh's account wherein higher learning rates are attached to cues which are good predictors (assuming that learning rates generalize to similar cues). 
However, a contrasting result has been reported in some experiments. For example, working with rats, Wilson, Boumphrey, and Pearce (1992) trained a tone as a conditioned reinforcer based upon pairing with food and trained a light as a predictor of the tone. In one group, they included some light-alone trials making the light a relatively poor predictor of the tone compared to another group in which the tone always occurred after the light. Following this training, conditioning to the light by presentation of light-food trials progressed more rapidly for the group which had the light alone trials. This result is consistent with the Pearce-Hall model, in which a poor predictor receives more processing than a good predictor and is therefore learned about more quickly.

These contrasting results (see also Griffiths, Johnson, \& Mitchell (2011) for a recent study and further discussion), which are based on indirect measures of attention, are relevant to the theoretical questions addressed (i.e., the conflicting predictions of the Mackintosh and Pearce-Hall models) but a change in learning performance per se does not make the nature of the attentional process explicit. At one level, more rapid learning about one stimulus than another must imply more attention, at least in the sense that the learning mechanism must be more effectively engaged for more rapid learning. However, more attention could be achieved in different ways, for example, greater active sampling of a stimulus or more effective processing of an already sampled stimulus. We therefore define direct measures of attention as those which serve to characterize the nature of attentional change in terms of the psychological mechanism employed to facilitate processing (Le Pelley et al., 2013).

Durlach and Mackintosh (1986) used only an indirect measure of attention but Wilson et al. (1992) used direct as well as indirect measures. Specifically, their direct measure of attention involved orienting responses to the light throughout the experiment. It was found that 
orientation towards the light (rearing, sniffing, forepaw contact) declined with trials whilst the light became established as a predictor of the tone but when the light-alone presentations began there was a dramatic increase in orientation to the light, indicating more attention coincident with the increase in predictive error produced by the light-alone trials. Thus, the increase in attention was manifest in overt stimulus sampling.

Studies with humans have also used direct and indirect measures of attention. Using a symptom-disease learning paradigm, Kruschke and Blair (2000) demonstrated reduced learning about cues which had been established as poor predictors during a blocking treatment (Kamin, 1969). Attention was then indirectly assessed by responses to compound cues which contained elements that had been associated with different outcomes. The choice of outcome indicated the strength of association and stronger associations were assumed to have arisen because of greater attention. The indirect measurement of attention used in this study does not provide detailed information about the underlying psychological process summarized under the heading of attention. In contrast, some human studies measured eye-movements concurrently with a learning task in order to see how this peripheral attentional process responded to stimuli with different predictive values (e.g., Kruschke, Kappenman, \& Hetrick, 2005; Wills, Lavric, Croft, \& Hodgson, 2007). Similar to Kruschke and Blair (2000), Kruschke et al. presented participants with cue compounds and defined an index of the relative strength of association by the choice of one outcome over another. However, rather than simply inferring any differences were due to attentional variation, they also observed shorter gaze durations to blocked cues than to controls. Similar results were obtained in another blocking experiment by Wills et al. (Experiment 2), showing shorter dwell times for a blocked, as compared to a control cue. Recently, Le Pelley et al. (2013) used a dot-probe methodology and observed faster responses to a target when the 
target appeared in a location cued by a predictive stimulus than when the target location was cued by a non-predictive stimulus, suggesting enhanced visual attention to the location of predictive cues. This cueing effect was only present when the target was presented within 250 ms of the cue suggesting an automatic attentional cueing effect.

Studies of eye-movements and visual attention during associative learning have therefore helped to make concrete the nature of the attentional change in learning. They suggest that in humans there are changes in stimulus sampling during learning, in agreement with animal studies (Wilson et al., 1992), and there are changes in visual attention. However, there is less information to document changes in the central processing of the sampled stimulus events. The Wills et al. study mentioned above also measured electroencephalogram event-related-potential differences between a redundant (blocked) and an informative stimulus which they interpreted as “enhanced visual discrimination" of the informative cue (Wills et al., 2007, p. 850).

The study recently reported by Livesey et al. (2009) is a further effort in this direction. Livesey et al. showed better predictors suffered less AB and provided support for the Mackintosh model. Moreover, AB provides additional information about the attentional change that takes place, beyond that involved with peripheral responses such as eye movements. AB is a deficit in reporting the second of two targets in rapid serial visual presentation (RSVP) of a stream of stimuli if the first target (designated T1) precedes the second target (T2) by a short duration of $200-500$ ms (Raymond, Shapiro, \& Arnell, 1992). AB may be considered to reflect the central processing of a stimulus sample rather than stimulus sampling and therefore changes in $\mathrm{AB}$ during learning may represent a fundamentally different kind of attentional change than those that have been demonstrated in studies of eye-movements or orientation responses. Success in reporting of $\mathrm{T} 2$ confirms the central processing of a stimulus beyond the change in stimulus 
sampling that can be determined with a peripheral measure such as increased gaze duration.

Therefore, in the current studies we wished to follow-up Livesey et al's (2009) investigation not only because their reported $\mathrm{AB}$ effect helps characterize the attentional change during learning, but also because the precise nature of the $\mathrm{AB}$ effect is of theoretical relevance. It has already been noted that Livesey et al.'s data supported the Mackintosh model over the Pearce-Hall model but it is important to emphasize that this is a single result which has not, to our knowledge, been replicated.

In Livesey et al. (2009), participants experienced a series of trials each of which consisted of an $\mathrm{AB}$ task embedded within a choice-reaction-time (CRT) task. Each trial began with an RSVP stream of letters in the centre of a computer screen. Immediately after the RSVP stream, participants had to indicate as rapidly as possible the location of a target stimulus (the CRT task), which was a filled circle appearing on the left or on the right-hand side of the screen. As soon as they had completed the CRT task, they were asked to report the identity of the two letters (T1 and T2) from the RSVP stream (the AB task). Critical to the design, a predictive relationship was established between some stimuli in the RSVP stream and the response requirement in the CRT task. T1 and T2 were red and thus stood out from the remaining white stimuli in the RSVP stream. Importantly, some of the T2 stimuli reliably predicted the location of the upcoming CRT target. Thus, if participants learned the relationship between those T2 stimuli and the CRT requirement, response times (RTs) on those CRT trials could be shorter than on other trials. Indeed, their participants did respond faster on the CRT trials when a predictive T2 stimulus had appeared. Consistent with previous studies, Livesey et al. found that participants had a deficit in correctly reporting the identity of T2 stimuli that occurred $200 \mathrm{~ms}$ after T1, an AB effect. 
Crucially, this deficit was less marked for those T2 stimuli which predicted the outcome of the CRT task.

Looking ahead to the results of the first of the two experiments to be reported below we did not find reduced $\mathrm{AB}$ to good predictors. In an attempt to understand why this might have been the case we speculated that observation of the AB learning effect may depend on the balance of two antagonistic attentional processes as suggested by hybrid attentional models. As described earlier, the Mackintosh and Pearce-Hall models both make use of the concept of prediction error in order to adjust learning rate. However, as shown in the equations of Figure 1, the Mackintosh model is based on relative prediction error whereas the Pearce-Hall model is based on absolute prediction error. In Figure $1, \lambda$ represents the value of the outcome on a learning trial and $V$, associative strength, represents the expectation. Equations 1 and 2 show how the models update $V$ on each trial whereas Equations 1a and 2a show how they make use of two distinct types of prediction error. In the Mackintosh model relative error is used to adjust the learning rate for a cue. Thus, cues which have small relative predictive error, i.e. relative to the combined predictive strength of all other cues present on a trial (as shown in Equation 1a: $\left.\left|\lambda-V_{a}\right|<\left|\lambda-\Sigma V_{x}\right|\right)$, will have their learning rate increased. In contrast, in the Pearce-Hall model, cues have their learning rate set to the absolute prediction error on the previous trial (as shown in Equation 2a: $\left.\left|\lambda^{n-1}-\Sigma V^{n-1}\right|\right)$. Therefore, a cue which occurs on a trial with an unexpected outcome will have a larger learning rate set on the next trial than will a cue which occurred on a trial with an expected outcome. These theoretical models thus capture two distinctly different computations that can be derived from the environmental inputs.

For the present discussion we note the distinction between absolute and relative prediction error and the fact that, based on these two models of attention in learning, the learning 
rate adjustment following a reduction in prediction error is likely to depend on whether the reduction is in absolute or relative error. Thus, we might expect a cue with small relative error could have its learning rate increased as could a cue with large absolute error. In such a scenario, assuming the learning rate is reflected in $\mathrm{AB}$, a comparison of $\mathrm{AB}$ between cues with different predictive values would not necessarily yield clear differences unless absolute or relative error was somehow dominant. Carrying this logic through, after failing to observe reduced $\mathrm{AB}$ to good predictors in Experiment 1, we changed the procedures in Experiment 2 in an attempt to highlight the smaller relative prediction error of the good predictors. In Experiment 2 we did observe reduced $\mathrm{AB}$ to the good predictors suggesting that relative prediction error is linked to reductions in $\mathrm{AB}$.

\section{Experiment 1}

\section{Method}

\section{Participants}

Second year psychology students at the University of Southampton were required to take part for a laboratory class. One hundred and thirty-nine participants (out of 153) gave written consent for their data to be used for further research and publication. From them, 35 participants were excluded (see Data Preparation and Analyses), leaving 104 participants for analyses. The mean age was 19 years and approximately one in five were male.

\section{Apparatus and Materials}

Computers were used for onscreen stimulus presentation and response recording. The experiment was programmed in Python using the PsychoPy extension (Peirce, 2007). The screen 
refresh rate was set at $60 \mathrm{~Hz}$. The modal stimulus display had a character in the screen centre and two green-outlined circles that flanked the character symmetrically on the left and right (Figure 2a). Eighteen characters (A, C, D, F, H, K, L, N, O, P, R, S, T, U, V, Y, +, and \#) were used; they appeared in red or green in normal Arial font. The height of a letter measured about 2.5 degrees of visual angle (dva) at a viewing distance of $50 \mathrm{~cm}$. Red and green were approximately photometrically equiluminant $(\sim 5.5 \mathrm{~cd} / \mathrm{m} 2)$. The radius of a circle was $4 \mathrm{dva}$ and the centre-to-centre distance between circles was 10 dva. One of the circles was filled in green in the choice response time (CRT) task (left or right circle). Stimuli were presented on a lavender background.

\section{Design and Procedure}

There were two parts. A CRT task was used in Part 1 to provide predictive training. An $\mathrm{AB}$ task was used in Part 2 to measure the $\mathrm{AB}$ effect respectively to predictive and nonpredictive stimuli established in the CRT task. In Livesey et al. (2009; Experiment 1 and Experiment 2 phase 1) the CRT learning task and AB task were run concurrently. We originally tried concurrent tasks but did not find any evidence for differential $\mathrm{AB}$ and in one experiment participants failed to show differential responding on the CRT learning task (Glautier \& Shih, unpublished). Livesey et al. found stronger differential AB for predictive and non-predictive stimuli when the AB task was separated from the CRT (Livesey et al., 2009; Experiment 2, phase 2). In the current studies we therefore separated the learning and $\mathrm{AB}$ tasks to minimize mutual interference. RSVP streams were presented in each phase but the task requirement (CRT or T1/T2 report) and the stimuli were varied across phases. Introductory instructions were displayed on the screen before each task began; each task took about 13 minutes to complete. 
During each task participants initiated each trial by pressing ' $Z$ ' and ' $M$ ' simultaneously when they were ready.

Both tasks began each trial with a $500 \mathrm{~ms}$ central, green fixation '+', followed by an RSVP stream of 14 characters -- beginning with '\#', then 12 different letters, and ending with '\#' (see Figure 2b). Each character remained in view for $100 \mathrm{~ms}$ and was immediately replaced by the next character. Each RSVP stream contained two red target letters (T1 and T2) in the AB task and one red letter cue in the CRT task. The remaining characters appeared in green. T2 and the cue letter always appeared as the third item from the end of the RSVP stream.

CRT task for predictive learning. During the CRT task, the two outline flanker circles remained on the screen from fixation to the end of the RSVP stream and one of them became filled in green when the RSVP stream ended; participants were to press ' $Z$ ' (or ' $M$ ') if the left (or right) circle was filled. Accuracy and response time (RT) were recorded for each trial.

The critical variable was the association between the identities of the red letter (T2) in the RSVP stream and designated choice responses. T2 had four possible identities $(\mathrm{C}, \mathrm{F}, \mathrm{H}$, and P); each was randomly assigned to one of the four (A, B, X, and Y) stimulus roles for each participant (see Table 1). On A->L trials, the correct response was left. On B->R trials, the correct response was right. On $\mathrm{X}->\mathrm{L} / \mathrm{R}$ and $\mathrm{Y}->\mathrm{L} / \mathrm{R}$ trials, the correct response was equally likely to be left or right. Random assignment to trial type meant that, for example, for one participant letter $\mathrm{C}$ could be used as the $\mathrm{A}$ cue, letter $\mathrm{F}$ as the $\mathrm{B}$ cue, letter $\mathrm{H}$ as the $\mathrm{X}$ cue, and letter $\mathrm{P}$ as the $\mathrm{Y}$ cue, whereas for the next participant $\mathrm{C}, \mathrm{F}, \mathrm{H}$, and $\mathrm{P}$ could be used as the $\mathrm{X}, \mathrm{A}$, B, and Y cues (respectively). Participants were told that some of the stimuli in the RSVP stream might enable them to predict the location of the circle and that they could improve their CRT performance by paying attention to the letters in the RSVP stream. 
Two practice trials using the non-predictive stimuli were presented before the 50 experimental blocks began. Each block contained four trials, one instance of each trial type given in Table 1. The order of trial types was independently randomized for each block. Feedback was provided immediately after each CRT response by displaying the RT for the current trial if the response was correct; otherwise, the correct response (e.g., 'M') was presented along with the current CRT display. After every two experimental blocks, the mean RT for correct responses of those two blocks of trials was presented along with the sentence, "Can you improve your speed?"

AB task. Immediately after the RSVP stream, participants received onscreen prompts to report $\mathrm{T} 1$ and $\mathrm{T} 2$ via the keyboard. They were instructed to make their best guess if they were unsure. Two variables were manipulated - T1-T2 lag and T2 identity. The lag was either 2 or 5 (i.e., T2 was the second or fifth item after T1; 200 or $500 \mathrm{~ms}$ ) to induce strong or weak (or no) AB on T2 (e.g. Shih \& Reeves, 2007). The T2 identity had five levels - C, F, H, P, and $\rho$. The letters $\mathrm{C}, \mathrm{F}, \mathrm{H}$, and $\mathrm{P}$ had special roles as either predictive or non-predictive cues in the CRT task and were only used as T2 items. T1 and the other RSVP stream items were selected randomly on each trial from those items not used for T2. The identity of $\rho$ was determined trial-by-trial by random selection from the remaining stimulus letters (i.e., a control trial type). Cross combination between the lag and T2 identity produced 10 experimental conditions.

The search task began with two practice trials of the control trial type, followed by 12 experimental blocks 10 of trials (one instance per condition). The presentation order of conditions was independently randomized for each block. Feedback about report accuracy (T1 and T2 collectively) was provided to the participants every two experimental blocks. 


\section{Data Preparation and Analyses}

CRT task. Prior to computing the mean RT for each condition, trials with incorrect responses or responses with RTs greater than 3000 ms were excluded. Next, for each participant, trials were recursively discarded where the RT fell outside the interval [M-3SD,M+3SD] where $\mathrm{M}$ and SD were the mean and standard deviation of the remaining RTs for that individual (e.g. Kim \& Cave, 1999). After this filtering procedure, the mean RT for each participant was obtained for the predictive $(\mathrm{A}->\mathrm{L}$ and $\mathrm{B}->\mathrm{R})$ and non-predictive $(\mathrm{X}->\mathrm{L} / \mathrm{R}$ and $\mathrm{Y}->\mathrm{L} / \mathrm{R})$ trials in the first and second halves of the CRT trials. For each participant at least 23 trials contributed to the means so defined.

AB task and participant exclusion. For the $\mathrm{AB}$ task, the dependent measures were accuracy of T1 report, $\mathrm{P}(\mathrm{T} 1)$, and accuracy of $\mathrm{T} 2$ report given that $\mathrm{T} 1$ had been correctly identified, $\mathrm{P}(\mathrm{T} 2 \mid \mathrm{T} 1)$. $\mathrm{P}(\mathrm{T} 1)$ and $\mathrm{P}(\mathrm{T} 2 \mid \mathrm{T} 1)$ were calculated for each participant, for the first and second halves of the $\mathrm{AB}$ task, for each of the three trial types (predictive, non-predictive, and control), and for each of the T1-T2 lags. Thus, for each cell defined by task-half, trial type, and lag, $\mathrm{P}(\mathrm{T} 1)$ was computed from 12 trials for the predictive and non-predictive trial types and 6 trials for the control trial type. Because some of our participants had small values of P(T1) they had very few trials for computing $\mathrm{P}(\mathrm{T} 2 \mid \mathrm{T} 1)$. Therefore to maximize the reliability of our measurements, we explored criteria for excluding participants based on the minimum number of trials available for computing $\mathrm{P}(\mathrm{T} 2 \mid \mathrm{T} 1)$, the variable of main interest. This number was set at five, yielding 35 participants excluded. Increasing this number to six (which was also the total number of trials for computing $\mathrm{P}(\mathrm{T} 1)$ for, e.g., first half-task, control trial type, at lag 2) would have resulted in exclusion of 91 participants. 
Statistical analyses. Analyses were carried out using R and associated packages

(Deepayan, 2008; Lawrence, 2012; R Development Core Team, 2012). ANOVAs were carried out using the "ez" package and follow-up t-tests were carried out with Welch's correction. Statistically significant differences are reported with $\alpha=.05$

\section{Results}

\section{Predictive learning in CRT task}

The group mean accuracy in the CRT task was $93 \%(S E=0.6)$. The grand mean for overall RT was $403 \mathrm{~ms}(S E=5.6)$ after an average of 2.0 trials per participant $(1.1 \%$ of correct responses) were discarded according to the filtering procedure. In order to determine whether participants learned the predictive value of the stimuli used in the CRT task the average RTs on the predictable outcome trials containing the two predictive cues $(\mathrm{CSp}$, the average of A and B, c.f. Table 1) and on the non-predictable outcome trials containing the two non-predictive cues (CSn, the average of $\mathrm{X}$ and $\mathrm{Y}$, c.f. Table 1) were obtained for each participant and for each of half of the CRT task. The means over all participants for each cue are shown in Figure 3, left panel. These data were subject to a 2 (phase: $1^{\text {st }}$ half vs. $2^{\text {nd }}$ half) $\times 2$ (Cue: CSp vs. CSn) repeated measures ANOVA. This produced a significant effect of phase, $F(1,103)=12.9, p$ $<.001$, indicating faster responses in phase 1 than in phase $2(\mathrm{M}=397 \mathrm{~ms}$ vs. $409 \mathrm{~ms})$. There was also an effect of cue, $F(1,103)=53.4, p<.001$, indicating faster responses to CSp than to CSn (see Figure 3 ) but there was no Phase $\times$ Cue interaction, $F(1,103)=1.34$. 


\section{Attentional blink}

Figure 4 shows $\mathrm{P}(\mathrm{T} 1)$ and $\mathrm{P}(\mathrm{T} 2 \mid \mathrm{T} 1)$ as a function of cue, phase, lag, measure, and learner status. Cue refers to the predictive role that the $\mathrm{T} 2$ item had fulfilled during the CRT task (CSp, average of $\mathrm{A}$ and $\mathrm{B}$; or $\mathrm{CSn}$, average of $\mathrm{X}$ and $\mathrm{Y}$ ) or to the control item type (CNT). Phase refers to the first and second half of the RSVP trials. Lag is the number of RSVP stream items between $\mathrm{T} 1$ and $\mathrm{T} 2$. Measure refers to $\mathrm{P}(\mathrm{T} 1)$ and $\mathrm{P}(\mathrm{T} 2 \mid \mathrm{T} 1)$. Learner is a between-subjects variable indicating whether or not the participants learned to discriminate between CSp and CSn in the CRT task. Participants who responded faster on the predictable outcome trials than on the nonpredictable outcome trials in the second phase of the CRT trials were classified as learners, the remainder were classified as non-learners. On this basis 75 out of 104 participants $(72 \%)$ were classified as learners. Learner was included as a factor because AB changes are predicated on learning in the CRT task and the original investigation of Livesey et al. showed that larger AB learning effects were present in participants who learned well. As can be seen from Figure 4 there was a strong $\mathrm{AB}$ effect -- $\mathrm{P}(\mathrm{T} 2 \mid \mathrm{T} 1)$ was much lower than $\mathrm{P}(\mathrm{T} 1)$ at lag 2 but this difference was not evident at lag 5. Additionally, the AB effect was stronger for the control trials (CNT in Figure 4), an effect likely to be due to the lower frequency with which the control stimuli were presented (Crebolder, Jolicoeur, \& McIlwaine, 2002).

A 3 (cue: CSp vs. CSn vs. CNT) x 2 (phase: 1 vs. 2) x 2 (lag: 2 vs. 5) x 2 (measure: P(T1) vs. P(T2|T1)) x 2 (learner status: non-learner vs. learner) mixed ANOVA was carried out on these data. All factors were within-subjects except for learner status. We were interested specifically in the possibility of the AB effect (i.e. a Lag x Measure interaction) varying with cue and learner status. The ANOVA produced a clear AB effect $\mathrm{F}(1,102)=48.9, p<.001$, which was subject to an interaction with cue, $\mathrm{F}(2,204)=3.08, p<.05$. There were no other interactions 
involving $\mathrm{AB}$. This result implies that the $\mathrm{AB}$ effect differs according to the cue. To determine the locus of the effect three-way ANOVAs (2 (cue: CS1 vs. CS2) x 2 (lag: 2 vs. 5) x 2 (measure: $\mathrm{P}(\mathrm{T} 1)$ vs. $\mathrm{P}(\mathrm{T} 2 \mid \mathrm{T} 1))$ ) were carried out separately for each pairwise combination of the three cue types. Each of these ANOVAs produced an AB effect, Fs(1,103)>114, ps<.001. In the two ANOVAs where CNT was analysed with CSp and with CSn there were Lag x Measure x Cue interactions, $\operatorname{Fs}(1,103)>13.2, p s<.001$, showing that the $\mathrm{AB}$ effect was larger for CNT than for either of CSp and CSn. In contrast, in the ANOVA analyzing CSp and CSn there was no Lag $\mathrm{x}$ Measure $\mathrm{x}$ Cue interaction, $\mathrm{F}(1,103)<1$, showing that the size of the $\mathrm{AB}$ effects did not differ for these two cues.

\section{Discussion}

Experiment 1 failed to show a predictive value effect on $\mathrm{AB}$ despite checks to demonstrate that three important criteria for a valid test were met. First, we obtained a clear AB effect overall indicating the general adequacy of our procedures. Second, AB was greater for control stimuli, $\rho$, than for either of CSp and CSn, an effect expected on the basis of the relative frequency of presentation of the different stimuli. Thus, our procedures detected $\mathrm{AB}$ and were sufficiently sensitive to replicate a standard result. Third, the failure to show differential AB effects for CSp and CSn cannot be explained in terms of participants failing to learn the CRT task adequately. We obtained strong learning effects overall and we did not observe a differential predictive value effect on $\mathrm{AB}$ for those classified as learners and non-learners based on their CRT task performance. However, we still cannot rule-out the possibility of a type 2 error. Although we did find a frequency of presentation effect on $A B$ this effect could be larger than a predictive value effect. Thus, it could be argued that our measurement of AB in the CSp and CSn 
conditions was not sensitive to small differences between conditions. Our strategy of excluding participants with fewer than five trials to estimate $\mathrm{P}(\mathrm{T} 2 \mid \mathrm{T} 1)$ in each experimental condition served to increase the reliability of our measurements but still, we might have found a small predictive value effect if we had used more trials.

On the basis of the distinction between absolute and relative prediction error, and the possibility that these may exert antagonistic effects on attentional processing (see Introduction), the failure to find significant differences between CSp and CSn on AB in Experiment 1 may have been the result of the balance of these two processes. To explain this in more detail consider an application of Equations 1a and 2a to the design of Experiment 1. For the predictable outcome trials the stimuli in the RSVP stream were the distinctive red CSp and the green background stimuli CSb. CSp was a good predictor of the outcome whereas the CSb stimuli were not. Therefore, applying Equation 1a, the relative prediction error for CSp was less than the relative prediction error for the other stimuli present i.e. $\left|\lambda-V_{C S p}\right|<\left|\lambda-V_{C S b}\right|$; and on that basis the Mackintosh model suggests that on the predictable outcome trials the learning rate for CSp would be increased whereas the learning rate for the CSb stimuli would be decreased. On the non-predictable outcome trials the stimuli in the RSVP stream were the distinctive red CSn and the green background stimuli CSb. The relative prediction errors for CSn and $\mathrm{CSb}$ were equal therefore the learning rates for these stimuli would be reduced on the nonpredictable outcome trials. Therefore, based on the Mackintosh model, we expected CSp to have a higher learning rate than CSn. Applying Equation 2a gives the absolute prediction error terms on the different trial types. The absolute error on the predictable outcome trials was smaller than on non-predictable outcome trials i.e. $\left|\lambda-V_{C S b}-V_{C S p}\right|<\left|\lambda-V_{C S b}-V_{C S n}\right|$ and since the Pearce-Hall model states that the learning rate is determined by the absolute error the learning 
rate for CSp would be set to a lower value than that for CSn. Thus, in Experiment 1, the PearceHall model predicts the opposite learning rate adjustments to the Mackintosh model and the two effects might well have cancelled each other out if these learning rate adjustments were reflected in $\mathrm{AB}$.

\section{Experiment 2}

To address this potential problem we used a design for the CRT learning task based on Haselgrove, Esber, Pearce, and Jones (2010; Experiment 1) and included two (instead of one) red cue letters in each RSVP stream of the CRT learning task (see Table 1). On the predictable outcome trials $(\mathrm{AV}->\mathrm{L}, \mathrm{AW}->\mathrm{L}, \mathrm{BV}->\mathrm{R}, \& \mathrm{BW}->\mathrm{R})$ cues $\mathrm{A}$ and $\mathrm{B}$ were predictive of the outcome in the same way as A and B predicted the outcome in Experiment 1 but in Experiment 2 additional cues $\mathrm{V}$ or $\mathrm{W}$ were included as distinctive red non-predictive comparison cues (CSr). Two distinctive red cues (X and $\mathrm{Y}$ ) were also included in the RSVP streams of the nonpredictable outcome trials (the XY->L/R trials). Applying Equations 1a and 2a to the design of Experiment 2 provides theoretical predictions for the outcome. As with Experiment 1, there were two trial types to consider, namely predictable and non-predictable outcome trials. On the predictable outcome trials the stimuli in the RSVP stream were the distinctive red CSp, a distinctive red non-predictive comparison cue CSr, and the green background stimuli CSb. CSp was a good predictor of the outcome whereas CSr and CSb were not. In terms of Equation 1a, the relative prediction error for CSp was less than the relative prediction error for the other stimuli present i.e. $\left|\lambda-V_{C S p}\right|<\left|\lambda-V_{C S b}-V_{C S r}\right|$; and on that basis the Mackintosh model suggests that the learning rate for CSp would be increased whereas the learning rate for the CSr stimuli would be reduced on the predictable outcome trials because $\left|\lambda-V_{C S r}\right|>\mid \lambda-V_{C S b}-$ 
$V_{C S p} \mid$. On the non-predictable outcome trials the stimuli in the RSVP stream were the two distinctive red CSn stimuli and the green background stimuli CSb. The relative prediction errors for the two CSn stimuli were both equal to the CSb stimuli and therefore the learning rates for these stimuli would be reduced on the non-predictable outcome trials. As a result, based on the Mackintosh model, we expect CSp to have a higher learning rate than both CSn and CSr. And, CSn should also have a higher learning rate than CSr. Mackintosh (1975) suggested that magnitude of the learning rate adjustment be proportional to the differences between the quantities on either side of the inequalities in Equation 1a. Then assuming $\left|\lambda-V_{C S r}\right|=$ $\left|\lambda-V_{C S n}\right|$ and $\left|\lambda-V_{C S b}-V_{C S p}\right|<\left|\lambda-V_{C S b}-V_{C S n}\right|$ the learning rate for CSr is decreased more than for CSn. Applying Equation 2a gives the absolute prediction error terms on the predictable and non-predictable outcome trials of Experiment 2. As with Experiment 1 the absolute error on predictable outcome trials was smaller than on non-predictable outcome trials i.e. $\left|\lambda-V_{C S b}-V_{C S p}-V_{C S r}\right|<\left|\lambda-V_{C S b}-V_{C S n}\right|$ and according to Equation 2 a this would be expected to produce a reduction in the learning rate for CSp and for CSr. In contrast, on the nonpredictable outcome trials, the learning rates for CSn would be increased.

The foregoing analysis shows that in Experiment 2, as with Experiment 1, we could expect that learning rate adjustments based on relative and absolute prediction error might cancel one another out. For example, CSp cues A and B still get salience increased on the basis of relative prediction error and decreased on the basis of absolute prediction error. However, in Experiment 2, A and B were accompanied by distinctive red non-predictive comparison cues $\mathrm{V}$ and W. These non-predictive comparison cues stood out from the distractors, resembling cues A and B in that they were red and occupied the same locations (counterbalanced across trials) in the RSVP stream. It was thus intended that the small relative prediction error for cues A and B 
would be highlighted, by juxtaposition of the predictive cues with non-predictive comparators, and should therefore drive attentional changes more strongly than the absolute prediction error. If attentional changes were driven entirely by relative error we would expect the rank ordering of cue saliences to be $\mathrm{CSp}>\mathrm{CSn}>\mathrm{CSr}$.

\section{Method}

Experiment 2 was the same as Experiment 1 except as follows. Another cohort of students took part and 169 participants gave consent for their data to be used for further research and publication Sixty participants were excluded using the same criterion as Experiment 1, leaving 109 participants for analyses.

CRT task. The RSVP stream for the CRT task included two, instead of one, red letters -$\mathrm{C} 1$ and $\mathrm{C} 2 . \mathrm{C} 1$ appeared as the sixth item in the RSVP stream and C2 as the third item from the end of the RSVP stream; thus, the C1-C2 lag was 600 ms. Six, instead of four, letters -- B, C, F, $\mathrm{H}, \mathrm{P}$, and $\mathrm{X}$-- were randomly assigned to one of the six stimulus roles for each participant (Table 1). Each block of CRT trials contained twelve trials; eight CSp trials and four CSn trials. The CSp trials comprised AV->L, AW- $>$ L, BV->R, and BW->R trials. Each exemplar appeared twice, once for each of the two possible cue orders in the RSVP stream. For example the AV trials would occur once with A appearing as $\mathrm{C} 1$ and $\mathrm{V}$ appearing as $\mathrm{C} 2$, and once with this order reversed. The four CSn trials comprised four presentations of XY->L/R, two of which had cue $X$ as $\mathrm{C} 1$ and $\mathrm{Y}$ as $\mathrm{C} 2$, and vice-versa for the other two trials. There were 16 experimental blocks of 12 CRT learning trials.

AB task. The AV, AW, BV, and BW trials introduced another cue type for the AB task. Cues $\mathrm{V}$ and $\mathrm{W}$ were designated non-predictive comparison cues $(\mathrm{CSr})$ to distinguish them from 
the non-predictive cues $\mathrm{X}$ and $\mathrm{Y}$. Thus, the T2 identity had seven levels -- B, C, F, H, P, X, and p. The cross-combination of T2 identity and T1-T2 lag ( 2 or 5) defined 14 conditions of an experimental block and there were 12 experimental blocks.

\section{Results}

\section{Predictive learning in CRT task}

The group mean accuracy in the CRT task was $97 \%(S E=0.2)$. The grand mean for overall RT was $414 \mathrm{~ms}(S E=3.8)$ after an average of 2.6 trials per participant $(1.4 \%$ of correct responses) were discarded according to the filtering procedure. As in Experiment 1, learning in the CRT task was assessed using a 2 (phase: $1^{\text {st }}$ half vs. $2^{\text {nd }}$ half) $\times 2$ (cue: CSp vs. CSn) repeated measures ANOVA. The AV, AW, BV, and BW trials were averaged for the CSp trials whereas the XY trials were averaged for CSn (c.f. Table 1). This produced qualitatively similar results to those observed in Experiment 1 (see Figure 3). There was a significant effect of phase and cue $F s(1,108)>6.87, p s<.05$, but no Phase x Cue interaction $F(1,108)<1$. Responses were faster in Phase 2 than in Phase 1 (412 vs. 420 ms) and faster on CSp than on CSn trials (see Figure 3), showing participants were anticipating the correct CRT response on the basis of the cues present in the RSVP stream.

\section{Attentional blink}

Figure 5 shows $\mathrm{P}(\mathrm{T} 1)$ and $\mathrm{P}(\mathrm{T} 2 \mid \mathrm{T} 1)$ as a function of cue, phase, lag, measure, and learner status. Cue refers to the predictive role that the T2 item had fulfilled in the CRT task (CSp, CSn, or CSr). Cues A and B were averaged for CSp, cues X and Y were averaged for CSn, and cues V and $\mathrm{W}$ were averaged for $\mathrm{CSr}$. As with Experiment 1, inspection of the figure indicates a strong 
AB effect. However, in Figure 5, it appears that CSp may have suffered less AB than the other CS cues, especially among the learners and in phase 1.

A 3 (cue: CSp vs. CSn vs. CSr) x 2 (phase: $1^{\text {st }}$ half vs. $2^{\text {nd }}$ half) x 2 (lag: 2 vs. 5) x 2 (measure: $\mathrm{P}(\mathrm{T} 1)$ vs. $\mathrm{P}(\mathrm{T} 2 \mid \mathrm{T} 1))$ x 2 (learner status: non-learner vs. learner) mixed ANOVA was carried out on these data. Seventy-one out of the 109 participants $(65 \%)$ were classified as learners. The ANOVA produced a strong overall AB effect, $\mathrm{F}(1,107)=94.1, p<.001$, but this was subject to a four-way interaction involving Cue $\mathrm{x}$ Phase, $\mathrm{F}(2,214)=3.29, p<.05$, and a five-way interaction involving Cue $\mathrm{x}$ Phase $\mathrm{x}$ Learner, $\mathrm{F}(2,214)=3.90, p<.05$. These higher order interactions indicate that the $\mathrm{AB}$ effect varied according to cue, phase, and learner status. To determine the precise nature of this interaction further mixed five-way ANOVAs were carried out for each pairwise combination of the three cue types ((cue: CS1 vs. CS2) x 2 (phase: $1^{\text {st }}$ half vs. $2^{\text {nd }}$ half) x 2 (lag: 2 vs. 5) x 2 (measure: P(T1) vs. P(T2|T1)) x 2 (learner status: non-learner vs. learner)). In all cases there was a highly significant $\mathrm{AB}$ effect $\operatorname{Fs}(1,107)>75.0, p_{s}<.001$. However, only in the analysis comparing CSp and CSn was this AB effect subject to further interactions involving cue and learner. In that analysis the five-way interaction remained significant, $\mathrm{F}(1,107)=8.18, p<.01$. Two four-way ANOVAs were then carried out, one for each of the two phases of trials. Both showed AB effects Fs(1,107)>54, ps<.001.

In the phase 1 four-way ANOVA the $\mathrm{AB}$ effect interacted with cue $\mathrm{F}(1,107)=4.23, p<.05$ indicating that the $\mathrm{AB}$ effect was larger for CSn than for CSp irrespective of learner status. Further clarification was obtained by calculation of AB scores, subtracting $\mathrm{P}(\mathrm{T} 2 \mid \mathrm{T} 1)$ from $\mathrm{P}(\mathrm{T} 1)$ at each lag, for CSp and CSn. These AB scores were smaller for CSp $(M=0.25)$ than for CSn $(\mathrm{M}=0.31)$ at lag 2, $\mathrm{t}(108)=2.11, p<.05$, but not at lag 5, $\mathrm{t}(108)=0.60 ; \mathrm{CSp} \mathrm{M}=0.03, \mathrm{CSn} \mathrm{M}=0.04$. 
Furthermore, at lag 2 CSp and CSn did not differ on P(T1), t(108)=0.47; CSp M=0.94 and CSn $\mathrm{M}=0.93$, but they did differ on $\mathrm{P}(\mathrm{T} 2 \mid \mathrm{T} 1), \mathrm{t}(108)=2.59, p<.05 ; \mathrm{CSp} \mathrm{M}=0.69$ and $\mathrm{CSn} \mathrm{M}=0.63$

For phase 2 the four-way ANOVA gave rise to a four-way interaction with AB, cue, and learner $F(1,107)=8.39, p<.01$. Final Cue $x$ Lag $x$ Measure ANOVAs carried out separately in phase 2 for the learners and non-learners revealed significant $A B$ effects for both groups, $\mathrm{F}(1,70)=63.1, p<.001$ and $\mathrm{F}(1,37)=68.3, p<.001$, respectively. In addition a significant Cue $\mathrm{x}$ Lag $\mathrm{x}$ Measure effect was found for the learners, $\mathrm{F}(1,70)=5.36, p<.05$, and a marginal Cue $\mathrm{x}$ Lag $\mathrm{x}$ Measure effect was found for the non-learners, $\mathrm{F}(1,37)=4.12, p=.05$; the size of the difference between cues on $\mathrm{AB}$ was greater among the learner group than amongst the non-learner group in phase 2. For the non-learners at lag $2 \mathrm{CSp}$ and CSn AB scores (P(T1)-P(T2|T1)) were not reliably different, $\mathrm{M}=0.28$ and $\mathrm{M}=0.26$ respectively; $\mathrm{t}(37)=0.66$. At lag 5 the $\mathrm{CSp}$ and $\mathrm{CSn} \mathrm{AB}$ scores showed a significant difference, $\mathrm{M}=0.015$ and $\mathrm{M}=0.067$ respectively; $\mathrm{t}(37)=2.0, p<.05$. Lag 5 comparisons of CSp and CSn on $\mathrm{P}(\mathrm{T} 1)$ and on $\mathrm{P}(\mathrm{T} 2 \mid \mathrm{T} 1)$ did not reach significance but the main contributing factor to the lag 5 effect on the $\mathrm{AB}$ scores was on $\mathrm{P}(\mathrm{T} 1)$ rather than on $\mathrm{P}(\mathrm{T} 2 \mid \mathrm{T} 1)$. At lag $5 \mathrm{CSp}$ and CSn did not differ reliably on $\mathrm{P}(\mathrm{T} 1)(\mathrm{CSp} \mathrm{M}=0.91$ and $\mathrm{CSn} \mathrm{M}=0.94)$ nor on $\mathrm{P}(\mathrm{T} 2 \mid \mathrm{T} 1)(\mathrm{CSp} \mathrm{M}=0.89$ and $\mathrm{CSn} \mathrm{M}=0.87) ; \mathrm{t}(37)=1.77$ and $\mathrm{t}(37)=0.38$ respectively. For the learners the AB scores were smaller for CSp $(\mathrm{M}=0.18)$ than for $\operatorname{CSn}(\mathrm{M}=0.25)$ at lag 2 , $\mathrm{t}(70)=2.30, p<.05$, but the difference was not reliable at lag 5, $\mathrm{t}(70)=0.04 ; \mathrm{CSp} \mathrm{M}=0.025, \mathrm{CSn}$ $\mathrm{M}=0.024$. Lag 2 comparisons of CSp and CSn on $\mathrm{P}(\mathrm{T} 1)$ and on $\mathrm{P}(\mathrm{T} 2 \mid \mathrm{T} 1)$ were significant in the case of $\mathrm{P}(\mathrm{T} 1)$ but not in the case of $\mathrm{P}(\mathrm{T} 2 \mid \mathrm{T} 1)$ so the main contributing factor to the lag 2 effect on AB scores was on $\mathrm{P}(\mathrm{T} 1)$ rather than on $\mathrm{P}(\mathrm{T} 2 \mid \mathrm{T} 1)$. At lag 2 CSp and CSn differed on $\mathrm{P}(\mathrm{T} 1)$ (CSp $\mathrm{M}=0.92$ and $\mathrm{CSn} \mathrm{M}=0.95)$ but not reliably on $\mathrm{P}(\mathrm{T} 2 \mid \mathrm{T} 1)(\mathrm{CSp} \mathrm{M}=0.74$ and $\mathrm{CSn} \mathrm{M}=0.70)$; $\mathrm{t}(70)=2.27, p<.05$, and $\mathrm{t}(70)=1.58$, respectively. 
The main finding from the analysis of the $\mathrm{AB}$ data from Experiment 2 was that there was less AB for CSp than for CSn during phase 1. We did not find this result in Experiment 1 and we considered that the difference between the experiments might have been due to the introduction of CSr in Experiment 2. This argument requires that the results in the two experiments do actually differ from one another and in order to examine this question an additional exploratory analysis was carried out on the combined data from Experiments 1 and 2. A 2 (cue: CSp vs. CSn) x 2 (phase: 1 st half vs. $2^{\text {nd }}$ half) x 2 (lag: 2 vs. 5) x 2 (measure: P(T1) vs. P(T2|T1)) x 2 (learner status: non-learner vs. learner) x 2 (Experiment: 1 vs. 2) mixed ANOVA showed a significant six-way interaction $\mathrm{F}(1,209)=4.01, p<.05$ meaning that the AB effect (Lag x Measure) differed according to cue, phase, learner status, and experiment. Combined with the main analyses these additional results confirm that Experiment 2 differed from Experiment 1 in that CSp suffered less $\mathrm{AB}$ than CSn in Experiment 2 but not in Experiment 1. In the main analysis of Experiment 1 a five-way ANOVA (2 (cue: CSp vs. CSn) x 2 (phase: 1 st half vs. $2^{\text {nd }}$ half) x 2 (lag: 2 vs. 5) x 2 (measure: P(T1) vs. P(T2|T1)) x 2 (learner status: non-learner vs. learner)) did not produce significant interactions involving cue and AB. In contrast, in the main analysis of Experiment 2, significant interactions involving cue and AB were found (see above for follow-up analyses).

\section{Discussion}

In Experiment 2, in contrast to Experiment 1, we observed reduced $\mathrm{AB}$ for predictive cues relative to non-predictive cues, thus replicating an effect originally reported by Livesey et al. (2009). The reduced AB effect was clear in the first phase of the RSVP trials where P(T2|T1) was larger at lag 2 for CSp stimuli than for CSn stimuli. However, the reduced AB effect in phase 2 was produced primarily by differences between CSp and CSn on P(T1) rather than on 
$\mathrm{P}(\mathrm{T} 2 \mid \mathrm{T} 1)$ and therefore not representative of an $\mathrm{AB}$ effect. A numerical difference in $\mathrm{AB}$ between CSp and CSr was also apparent among the learners in phase 1 (Figure 5) but this was not pursued because the overall ANOVA involving these cues failed to produce relevant significant results. The primary modification introduced for Experiment 2 was the inclusion of two red cues in the RSVP streams. The aim of this modification was to highlight the smaller relative prediction error for the CSp stimuli by providing an explicit non-predictive comparator cue, CSr. According to the relative predictive error principle encapsulated in the Mackintosh (1975) model any non-predictive comparison cue should result in an increased learning rate for the predictive cues (c.f. Introduction and Equation 1a) and the RSVP streams used Experiments 1 and 2 did in fact contain multiple cues (the green distractor elements, $\mathrm{CSb}$ ) which could have served as comparators for the predictive cues. However, we suggest that these distractors may not have been effective comparators because they differed in colour, frequency, and location in the RSVP streams from CSp. The CSr cues, on the other hand, were matched with CSp in these respects and we suggest that this facilitated a comparison between the predictive values of CSp and CSr, which increased the salience of the predictive CSp cues.

\section{General Discussion}

In the current studies we used $\mathrm{AB}$ as a specific direct measure of attention to stimuli that had different predictive values in a CRT learning task. CSp stimuli allowed performance enhancing predictions to be made in the CRT task whereas CSn and CSr stimuli did not. According to Mackintosh (1975) stimuli with small relative prediction errors should have their learning rates increased. According to Pearce and Hall (1980) stimuli with large absolute prediction errors should have their learning rates increased. Learning rate parameters are 
frequently interpreted in terms of stimulus salience with high salience stimuli commanding more attention than low salience stimuli. Previous studies mentioned in the introduction (e.g. Le Pelley, Vadillo, \& Luque, 2013; Wills, Lavric, Croft, \& Hodgson, 2007; Experiment 2; Wilson, Boumphrey, \& Pearce, 1992) have shown behavioural responses indicative of increased attention (eye-movements, orienting responses, visual attention) in response to both kinds of prediction error and hybrid theories of attention in associative learning assert that both kinds of prediction error play a role (Le Pelley, 2004; Pearce \& Mackintosh, 2010). The work of Livesey et al. (2009) appeared to show that good predictors had increased attention on an AB measure. This indicates preferential central processing of a stimulus rather than increased attention in terms of stimulus sampling. Predictive cues suffered less AB than non-predictive cues, an important result that requires replication and further exploration. In Experiment 1 we found that both predictive and non-predictive cues were equivalent in $\mathrm{AB}$ but in Experiment 2 we found that predictive cues suffered less AB than non-predictive cues. We suggest that the difference between the results of the two experiments was due to the inclusion of distinctive non-predictive comparison cues into the RSVP streams of Experiment 2 and that this increased the influence of relative prediction error in the production of attentional change. Some implications and limitations of this conclusion are addressed below.

In Livesey et al. (2009) predictive and non-predictive stimuli differed on both relative and absolute prediction error. The predictive stimuli had smaller relative error and occurred on trials with smaller absolute prediction error; they were better predictors than the other stimuli appearing in the same RSVP streams (T1 and distractor stimuli) and they occurred on trials for which the outcome could be predicted. The non-predictive stimuli had the same relative error as other stimuli in their RSVP streams and occurred on trials for which the outcome could not be 
predicted. Here we are not arguing that the smaller absolute error on the predictable outcome trials caused the reported reduction in $\mathrm{AB}$, although this cannot be ruled out. We merely wish to emphasize the point we made earlier that the attentional response has been hypothesized to be sensitive to both type of prediction error and the observed outcome in any particular experiment is likely to be the result of the balance of two processes. In Experiment 1 we did not observe reduced $\mathrm{AB}$ for predictive stimuli despite adequate learning and $\mathrm{AB}$ procedures, and stringent selection of participants from a large sample to maximize the reliability of our AB measurements. We conjecture that the larger absolute prediction error for our non-predictive stimuli may have contributed to this. At least, according to the Pearce-Hall model, absolute prediction error on those trials should have served to maintain attention to the non-predictive stimuli. However, we also hypothesized that the small relative prediction error for the predictive stimuli was underweighted. In Experiment 2 we attempted to test this by increasing the salience of relative prediction error, including a salient non-predictive comparison cue in the RSVP streams alongside the predictive cue. We maintained an absolute prediction error component between predictable and non-predictable outcome trials, our $\mathrm{AB}$, and participant sampling procedure, whereupon we observed the expected difference in $\mathrm{AB}$ between predictive stimuli and non-predictive stimuli. This result indicates the importance of a salient relative prediction error component for reducing $\mathrm{AB}$.

In addition to providing an indication of potential difficulties in obtaining a predictive learning effect on $\mathrm{AB}$, and a solution involving increasing the salience of non-predictive comparison stimuli, our studies confirm that $\mathrm{AB}$ changes can occur during learning. Our interest in this effect was motivated by the possibility of obtaining a more specific characterization of attentional changes during learning than can be obtained using indirect measures. Already it was 
known that stimulus sampling and visual attention changes can occur (e.g. Kruschke,

Kappenman, \& Hetrick, 2005; Le Pelley et al., 2013; Wills et al., 2007; Wilson et al., 1992) and

the study of Wills et al. indicated central changes in stimulus processing as suggested in ERP measures. The work of Livesey et al. (2009) and the current work on AB are additional steps to describe the underlying changes in central cue processing that can occur during learning. Subject to further replication and exploration we interpret the observed predictive learning effect on $\mathrm{AB}$ in terms of changes in top-down salience of predictive and non-predictive cues. Cognitive models of $\mathrm{AB}$ propose that top-down salience either maintains the strength of an activated T2 representation over an interval during the processing of $\mathrm{T} 1$ or that top-down salience determines resource allocation in working memory. For example, in the processing pipeline of the Attention Cascade Model of Shih (2008) information strength decays whilst a stimulus is queued to enter a consolidation processor. At short T1-T2 lags there is a longer queuing time for T2, due to processing of $\mathrm{T} 1$, therefore $\mathrm{T} 2$ is weaker on entering the processor, and has a smaller probability of being consolidated for identification. The initial strength of a stimulus is determined by activation of LTM traces when the stimulus is presented and activation is greater for stimuli with higher top-down salience. With respect to the current investigation, relative to CSp, CSn, and CSr, top-down salience for the control stimuli was expected to be lower because they served as targets less frequently (Figures 4 and 5 show much more AB for CNT stimuli). In addition, in the first phase of the RSVP trials for frequency matched stimuli CSp and CSn, the increased topdown salience of CSp was evident in the fact that CSp had higher chance of either exceeding the response threshold or being successfully consolidated than CSn (Figure 5), as indicate by $\mathrm{P}(\mathrm{T} 2 \mid \mathrm{T} 1)$ values. CSr showed a similar pattern to CSn amongst the learners in phase 1 but the overall analysis of CSr did not reach statistical significance. 
Although the difference between CSp and CSn on AB in Experiment 2 was clear some comments on the methodology and theoretical interpretation are warranted. We attribute the difference in the results of Experiments 1 and 2 to the increased weighting of relative prediction error consequent to the introduction of distinctive comparator stimuli in the RSVP stream. In terms of theoretical interpretation, assuming that relative error adjustments dominated the outcomes, it was expected on the basis of the analysis offered in the introduction to Experiment 2 that the rank ordering of the learning rates of the stimuli in Experiment 2 would be $\mathrm{CSp}>\mathrm{CSn}>\mathrm{CSr}$. Our results only partially confirm this, in that we only found a significant difference on AB between CSp and CSn. At face value this is incompatible with the Mackintosh model but the problem may not be a serious one because limitations are normally imposed on the extent to which learning rate adjustments are made (e.g. Le Pelley, 2004). Thus, it might be supposed that the learning rates for both CSn and CSr were adjusted to a lower limiting value, hence their equivalence. However, additional explanation is still required for the failure to see a significant difference between CSp and CSr. Again, this issue may not be fatal for the framework under consideration because numerically, we did obtain results consistent with the hypothesized learning rate rankings for learners in phase 1 (Figure 5). P(T2|T1) for CSp was more than 2.2 standard error units higher than for either CSn or CSr for the learners in phase 1. However, it is necessary to exercise some caution here because the overall ANOVA tell us that whilst the AB difference between CSp and CSn was significant the difference between CSp and CSr was not. Nevertheless, the data do not run strongly against the view that the relative prediction error model is a good candidate for explaining the observations, at least amongst learners. A further problem thrown-up by the comparison of CSp and CSn concerns the confounding of relative and absolute prediction error. The methodology of Experiment 2 may 
have highlighted relative prediction error but it did not disentangle the two distinct error components associated with these two cues. However, we argue that the confound of relative and absolute prediction error inherent in the comparison of CSp and CSn was present in both experiments, therefore something else must be held to account for the different results.

Although we attribute the observed difference between experiments to the introduction of CSr in Experiment 2 it is appropriate to mention three alternative explanations for the difference between the experiments ${ }^{1}$. First, there is the possibility that there was a difference between the participant populations in the two experiments. On the surface this seems somewhat unlikely given that we had the same sampling frame $\left(2^{\text {nd }}\right.$ year psychology laboratory class $)$ differing only in time of application. However, this could only be effectively ruled out by a within-experiment replication. Second, in Experiment 1 there was a shift from one to two red targets in the RSVP streams between the learning and $\mathrm{AB}$ tasks, whereas in Experiment 2 there was greater consistency between the tasks. This might have introduced an additional cognitive load in the AB task of Experiment 1 which could have somehow masked AB differences between cues with different predictive values. Third, there is some evidence that the learning task was more difficult in Experiment 2. Seventy-two percent of participants in Experiment 1 were classified as learners whereas only $65 \%$ of participants in Experiment 2 were so classified. In addition, the difference between response times for CSp and CSn in the CRT task was greater in Experiment 1 than in Experiment 2. This raises the possibility that increasing task difficulty would be sufficient to produce selective attention to CSp and that under conditions of lower task difficulty such a 'strategic' shift in attention does not occur. Our experiments do not allow us to make a strong judgment on such a possibility but we do note that the non-learners, who could reasonably be speculated to find the task more difficult than the learners, have the most to gain from strategic

\footnotetext{
${ }^{1}$ We are grateful to reviewers for suggesting these possibilities.
} 
allocation of attention. Despite this, the differences between cues was not apparent for the two groups and the original research on the AB learning effect suggests the opposite pattern - i.e. non-learners are, if anything, less likely to show the AB learning effect (Livesey et al., 2009). In summary, an attentional model of learning which suggests more attention to good than to poor predictors was supported in Experiment 2 (Mackintosh, 1975). However, this effect only appeared when steps were taken to increase the salience of relative prediction error by including salient non-predictive comparison stimuli in the RSVP streams containing the predictive cues. We interpret the AB learning effect in terms of increases in the top-down salience of predictive stimuli so that they are more likely to be available for report. In models of $\mathrm{AB}$ such as the Attention Cascade Model of Shih (2008) increases in top-down salience result in a greater likelihood that an RSVP stream target stimulus will either exceed a response threshold for report or be sufficiently salient to survive delayed entry to the consolidation processor. 
Table 1

Designs for Experiments 1 and 2

\begin{tabular}{cc}
\hline Experiment 1 & Experiment 2 \\
\hline $\mathrm{A}->\mathrm{L}$ & $\mathrm{AV}->\mathrm{L}$ \\
$\mathrm{X}-\mathrm{L} / \mathrm{R}$ & $\mathrm{AW}-\mathrm{L}$ \\
$\mathrm{Y}->\mathrm{L} / \mathrm{R}$ & $\mathrm{BV}->\mathrm{R}$ \\
& $\mathrm{BW}->\mathrm{R}$ \\
\hline
\end{tabular}

Note. The '->' indicates the response requirement in the presence of a particular cue. For example A->L means left response required in presence of cue A. In Experiment 2 the order of the cues within the RSVP stream was counterbalanced so that, for example, on AV->L trials cue A appeared first as often as cue V appeared first. For trial types with an L/R response requirement responses $\mathrm{L}$ and $\mathrm{R}$ were 'correct' equally often i.e. the outcome was unpredictable. 


\section{Figure Captions}

Figure 1. Formal models of learning. The Mackintosh model adjusts associative strength of a cue $\left(V_{a}\right)$ trial by trial based on an error term for that cue $\left(\lambda-V_{a}\right)$ as indicated in Equation 1 . The cue's learning rate parameter $\alpha_{a}$ is adjusted on the basis of a comparison of the error for that cue with the error for all other cues $\left(\lambda-\Sigma V_{x}\right)$ present on the trial (Equation 1a). In the Pearce-Hall model there is no explicit error term for the adjustment of a cue's associative strength (Equation 2). Instead, the absolute error term from the previous trial $\left|\lambda^{n-1}-\Sigma V^{n-1}\right|$ is used to adjust $\alpha_{a}$ (Equation 2a). $\Sigma V^{n-1}$ represents the combined associative strength of all the cues present on the previous trial.

Figure 2. Stimuli and procedure. (a) A typical stimulus display for CRT predictive learning task and the RSVP search task. For the choice response time (CRT) task, the middle character was replaced with a '+' sign; and the left or right circle was filled in green. Degrees of visual angle were based on a viewing distance of $50 \mathrm{~cm}$. (b) Schematic illustration of frames presented during each rapid serial visual presentation trial in the AB task for central letters in stimulus displays. Note that the two green outline circles remained on the screen throughout each RSVP stream. $\mathrm{D}=$ distractors; $\mathrm{T} 1=$ first target; $\mathrm{T} 2=$ second target. $\mathrm{T} 1$ and $\mathrm{T} 2$ appeared in red, while distractors in green. The stimulus onset asynchrony in an RSVP stream was $100 \mathrm{~ms}$ with no blank interval between stimuli.

Figure 3. Mean response time during the CRT predictive learning task for each experiment as a function of trial type. $\mathrm{CSp}=$ predictable outcome trials, $\mathrm{CSn}=$ non-predictable outcome trials. Vertical bars indicate the $S E$ of the means. 
Figure 4. RSVP task performance in Experiment 1 as function of cue, phase, lag, measure, and learner status. $\mathrm{CSp}=$ predictive cues, $\mathrm{CSn}=$ non-predictive cues, $\mathrm{CNT}=\rho$ cues. Vertical bars indicate the $S E$ of the means.

Figure 5. RSVP task performance in Experiment 2 as function of cue, phase, lag, measure, and learner status. $\mathrm{CSp}=$ predictive cues, $\mathrm{CSn}=$ non-predictive cues, $\mathrm{CNT}=\rho$ cues, $\mathrm{CSr}=$ nonpredictive reference cues. Vertical bars indicate the $S E$ of the means. 


\section{Mackintosh model}

Associative strength

$$
\Delta V_{a}=\alpha_{a} \beta\left(\lambda-V_{a}\right)
$$

Learning rate adjustment

$$
\Delta \alpha_{a} \begin{cases}>0 & \left|\lambda-V_{a}\right|<\left|\lambda-\Sigma V_{x}\right| \\ <0 & \left|\lambda-V_{a}\right| \geq\left|\lambda-\Sigma V_{x}\right|\end{cases}
$$

\section{Pearce-Hall model}

Associative strength

$$
\Delta V_{a}=S_{a} \alpha_{a} \lambda
$$

Learning rate adjustment

$$
\alpha_{a}^{n}=\left|\lambda^{n-1}-\Sigma V^{n-1}\right|
$$


a.

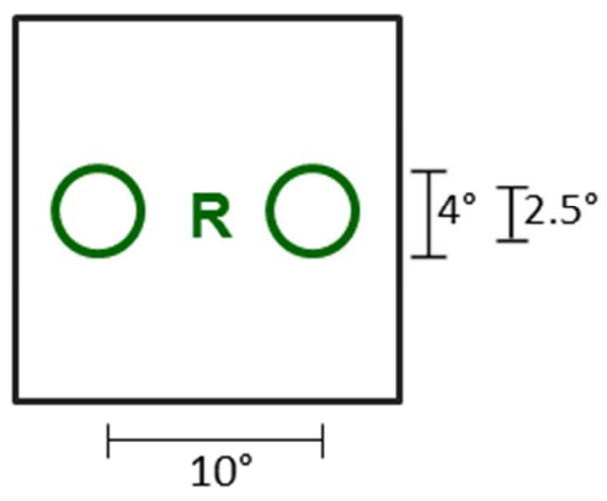

b.

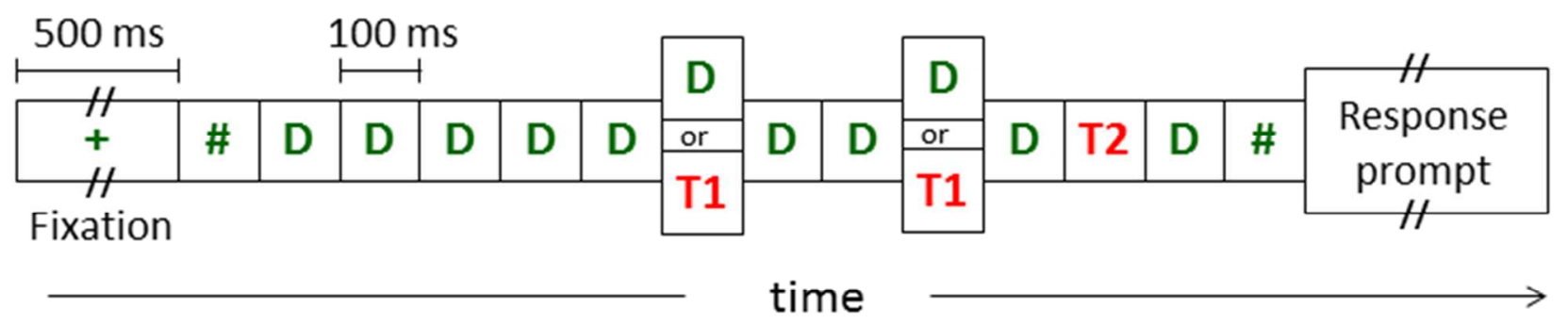




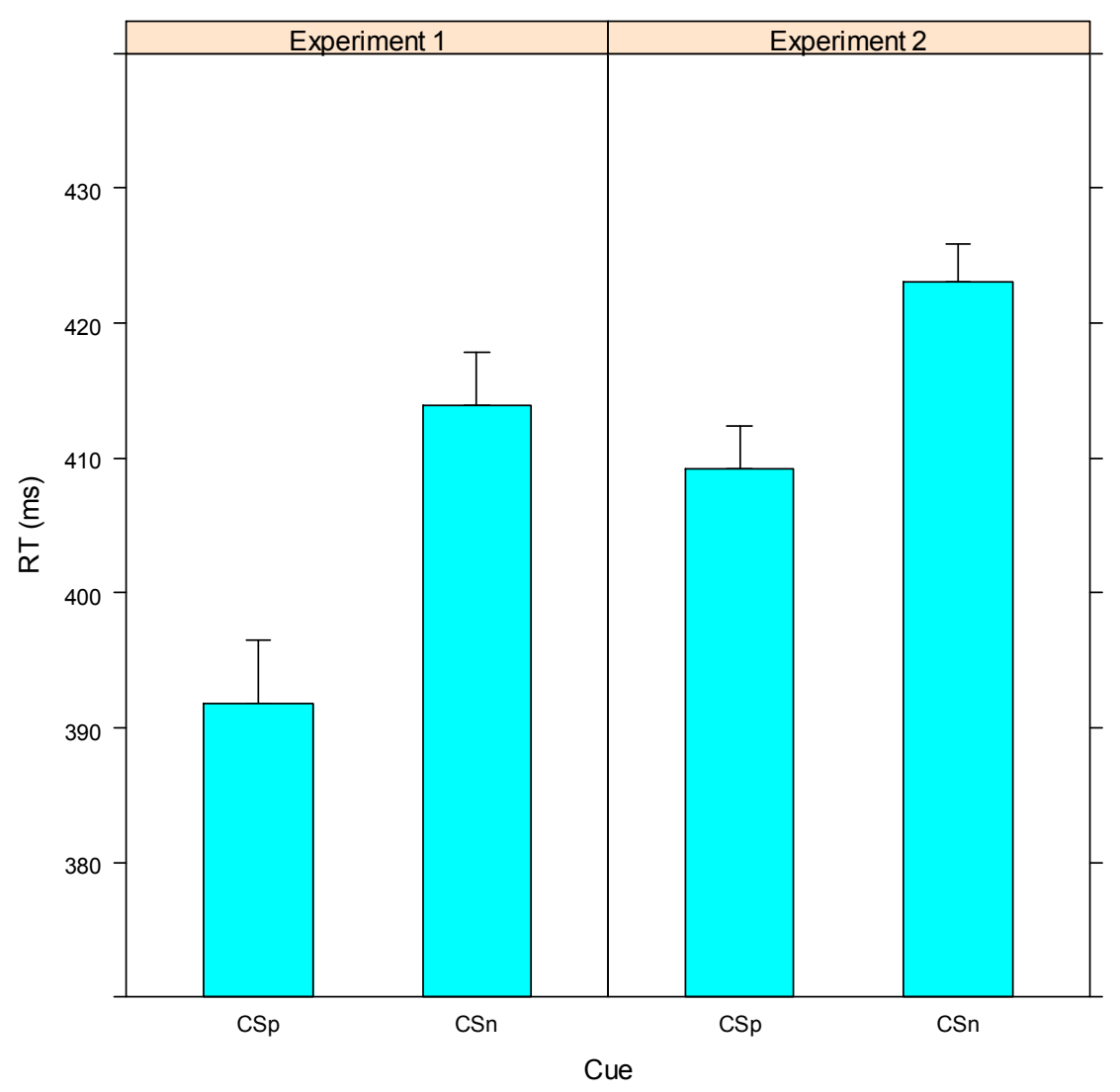



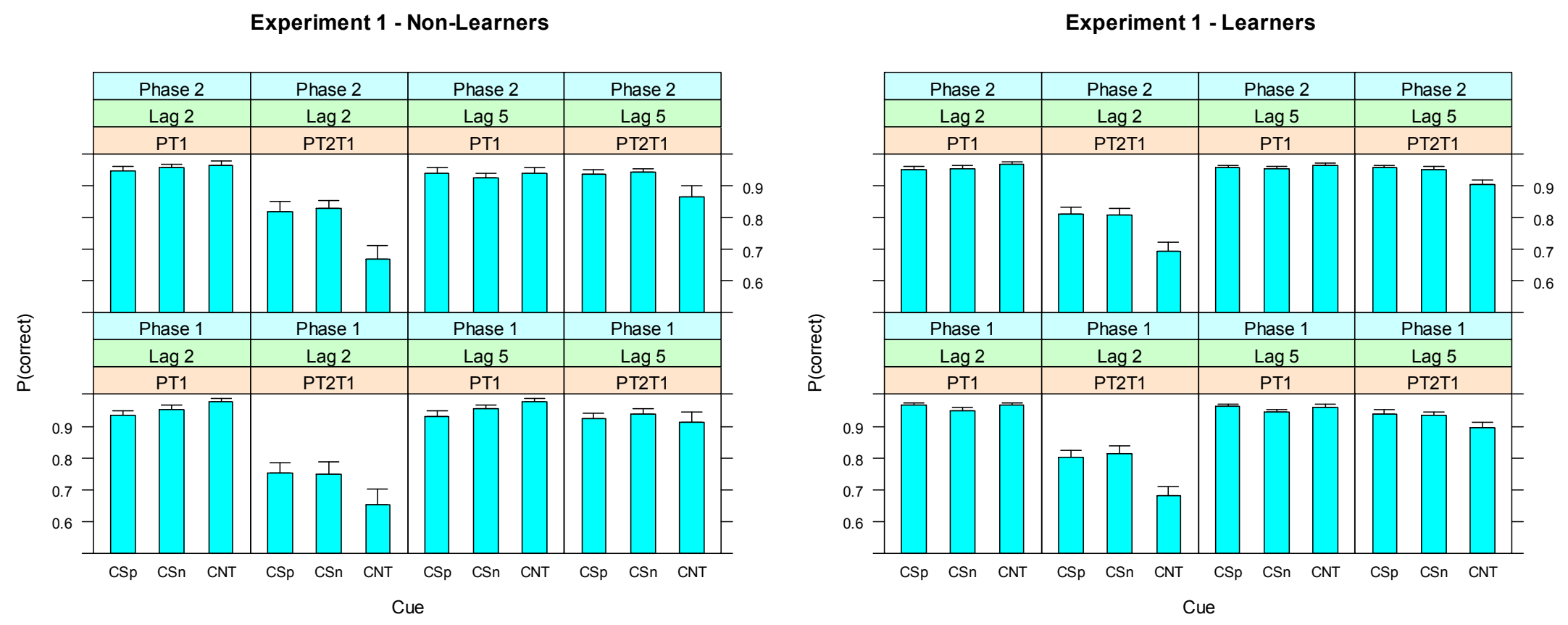

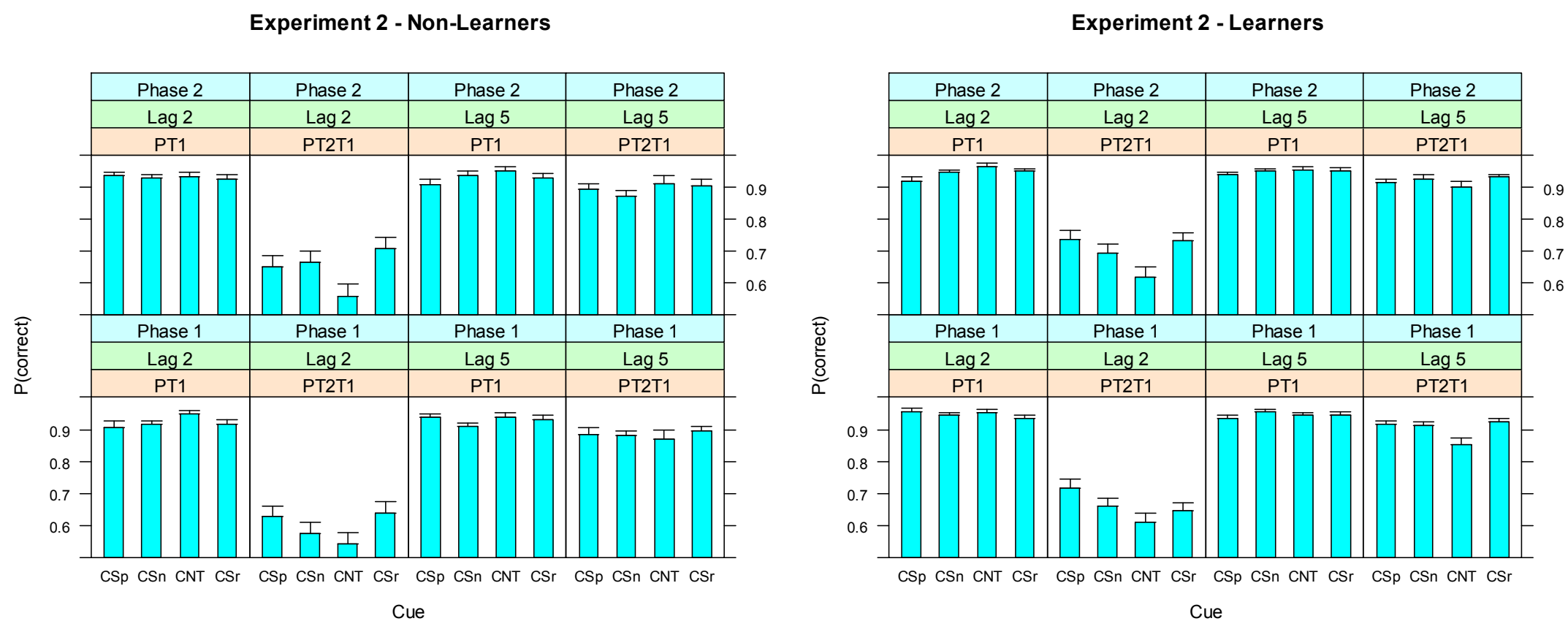
References

\author{
Crebolder, J. M., Jolicoeur, P., \& McIlwaine, J. D. (2002). Loci of signal probability effects and \\ of the attentional blink bottleneck. Journal of Experimental Psychology-Human \\ Perception and Performance, 28(3), 695-716. doi: 10.1037//0096-1523.28.3.695 \\ Deepayan, S. (2008). Lattice: Multivariate Data Visualization with R. New York: Springer. \\ Retrieved from http://lmdvr.r-forge.r-project.org
}

Durlach, P. J., \& Mackintosh, N. J. (1986). Transfer of Serial Reversal-Learning in the Pigeon. Quarterly Journal of Experimental Psychology Section B-Comparative and Physiological Psychology, 38(1), 81-95.

Griffiths, O., Johnson, A. M., \& Mitchell, C. J. (2011). Negative Transfer in Human Associative Learning. Psychological Science, 22(9), 1198-1204. doi: 10.1177/0956797611419305

Haselgrove, M., Esber, G. R., Pearce, J. M., \& Jones, P. M. (2010). Two Kinds of Attention in Pavlovian Conditioning: Evidence for a Hybrid Model of Learning. Journal of Experimental Psychology-Animal Behavior Processes, 36(4), 456-470.

Kamin, L. J. (1969). Predictability, surprise, attention and conditioning. In B. A. Campbell \& R. M. Church (Eds.), Punishment and Aversive Behavior (pp. 279-296). New Jersey: Prentice Hall.

Kim, M. S., \& Cave, K. R. (1999). Top-down and bottom-up attentional control: On the nature of interference from a salient distractor. Perception \& Psychophysics, 61(6), 1009-1023.

Kruschke, J. K., \& Blair, N. J. (2000). Blocking and backward blocking involve learned inattention. Psychonomic Bulletin \& Review, 7(4), 636-645. 
Kruschke, J. K., Kappenman, E. S., \& Hetrick, W. P. (2005). Eye gaze and individual differences consistent with learned attention in associative blocking and highlighting. Journal of Experimental Psychology-Learning Memory and Cognition, 31(5), 830-845.

Lawrence, M. A. (2012). ez: Easy analysis and visualization of factorial experiments (Version R package version 3.0-1). Retrieved from http://CRAN.R-project.org/package=ez

Le Pelley, M. E. (2004). The role of associative history in models of associative learning: A selective review and a hybrid model. Quarterly Journal of Experimental Psychology Section B-Comparative and Physiological Psychology, 57(3), 193-243.

Le Pelley, M. E., Vadillo, M., \& Luque, D. (2013). Learned Predictiveness Influences Rapid Attentional Capture: Evidence From the Dot Probe Task. Journal of Experimental Psychology-Learning Memory and Cognition, 39(6), 1888-1900. doi: 10.1037/a0033700

Livesey, E. J., Harris, I. M., \& Harris, J. A. (2009). Attentional Changes During Implicit Learning: Signal Validity Protects a Target Stimulus From the Attentional Blink. Journal of Experimental Psychology-Learning Memory and Cognition, 35(2), 408-422.

Mackintosh, N. J. (1975). Theory of Attention - Variations in Associability of Stimuli with Reinforcement. Psychological Review, 82(4), 276-298.

Pearce, J. M., \& Hall, G. (1980). A Model for Pavlovian Learning - Variations in the Effectiveness of Conditioned but Not of Unconditioned Stimuli. Psychological Review, $87(6), 532-552$.

Pearce, J. M., \& Mackintosh, N. J. (2010). Two theories of attention: A review and a possible integration. In M. E. Le Pelley \& C. J. Mitchell (Eds.), Learning and Attention. Oxford: Oxford University Press. 
Peirce, J. W. (2007). PsychoPy - Psychophysics software in Python. Journal of Neuroscience Methods, 162(1-2), 8-13. doi: DOI 10.1016/j.jneumeth.2006.11.017

R Development Core Team. (2012). R: A language and environment for statistical computing. Vienna, Austria: R Foundation for Statistical Computing. Retrieved from http://www.Rproject.org/

Raymond, J. E., Shapiro, K. L., \& Arnell, K. M. (1992). Temporary Suppression of Visual Processing in an Rsvp Task - an Attentional Blink. Journal of Experimental PsychologyHuman Perception and Performance, 18(3), 849-860.

Shih, S. I. (2008). The attention cascade model and attentional blink. Cognitive Psychology, $56(3), 210-236$.

Shih, S. I., \& Reeves, A. (2007). Attentional capture in rapid serial visual presentation. Spatial Vision, 20(4), 301-315.

Wills, A. J., Lavric, A., Croft, G. S., \& Hodgson, T. L. (2007). Predictive learning, prediction errors, and attention: Evidence from event-related potentials and eye tracking. Journal of Cognitive Neuroscience, 19(5), 843-854.

Wilson, P. N., Boumphrey, P., \& Pearce, J. M. (1992). Restoration of the Orienting Response to a Light by a Change in Its Predictive Accuracy. Quarterly Journal of Experimental Psychology Section B-Comparative and Physiological Psychology, 44B(1), 17-36. 